\title{
Colored Simultaneous Geometric Embeddings ${ }^{\star}$
}

\author{
U. Brandes ${ }^{1}$, C. Erten ${ }^{2}$, J. Fowler ${ }^{3}$, F. Frati ${ }^{4}$, M. Geyer ${ }^{5}$, \\ C. Gutwenger ${ }^{6}$, S. Hong ${ }^{7}$, M. Kaufmann ${ }^{5}$, S. G. Kobourov ${ }^{3}$, G. Liotta ${ }^{8}$, \\ P. Mutzel ${ }^{6}$, and A. Symvonis ${ }^{9}$ \\ 1 Department of Computer \& Information Science, University of Konstanz \\ ulrik.brandes@uni-konstanz.de \\ 2 Department of Computer Science, Isik University \\ cesim@isikun.edu.tr \\ 3 Department of Computer Science, University of Arizona \\ \{jfowler, kobourov\}@cs.arizona.edu \\ 4 Department of Computer Science, University of Roma Tre \\ frati@dia.uniroma3.it \\ 5 Wilhelm-Schickard-Institute of Computer Science, University of Tübingen \\ \{geyer, mk\}@informatik.uni-tuebingen.de \\ 6 Department of Computer Science, University of Dortmund \\ \{petra.mutzel, carsten.gutwenger\}@cs.uni-dortmund.de \\ 7 NICTA Ltd. and School of Information Technologies, University of Sydney \\ seokhee.hong@nicta.com.au \\ 8 School of Computing, University of Perugia \\ liotta@diei.unipg.it \\ 9 School of Applied Math. \& Phys. Sciences, National Technical University of Athens \\ symvonis@math.ntua.gr
}

\begin{abstract}
We introduce the concept of colored simultaneous geometric embeddings as a generalization of simultaneous graph embeddings with and without mapping. We show that there exists a universal pointset of size $n$ for paths colored with two or three colors. We use these results to show that colored simultaneous geometric embeddings exist for: (1) a 2-colored tree together with any number of 2-colored paths and (2) a 2-colored outerplanar graph together with any number of 2-colored paths. We also show that there does not exist a universal pointset of size $n$ for paths colored with five colors. We finally show that the following simultaneous embeddings are not possible: (1) three 6-colored cycles, (2) four 6-colored paths, and (3) three 9-colored paths.
\end{abstract}

\section{Introduction}

Visualizing multiple related graphs is useful in many applications, such as software engineering, telecommunications, and computational biology. Consider the case where a pair of related graphs is given and the goal is to visualize them so as to compare the two, e.g., evolutionary trees obtained by different algorithms. When visually examining relational information, such as a graph structure, viewers construct an internal model called the

\footnotetext{
* Work on this paper began at the BICI Workshop on Graph Drawing, held in Bertinoro, Italy in March 2006.
} 
mental map, for example, using the positions of the vertices relative to each other. When viewing multiple graphs the viewer has to reconstruct this mental map after examining each graph and a common goal is to aid the viewer in this reconstruction while providing a readable drawing for each graph individually. Simultaneous embeddings [4] aid in visualizing multiple relationships between the same set of objects by keeping common vertices and edges of these graphs in the same positions.

A simultaneous geometric embedding is a generalization of the traditional planar graph embedding problem, where we look for a common embedding of multiple graphs defined on the same vertex set. We omit the "geometric" clarification in the rest of the paper as we only consider straight-line drawings. There are two main variations of the problem. In simultaneous embedding with mapping the embedding consists of plane drawings for each of the given graphs on the same set of points, with corresponding vertices in the different graphs placed at the same point. In simultaneous embedding without mapping the embedding consists of plane drawings for each of the given graphs on the same set of points, where any vertex can be placed at any of the points in the point set.

Restricted subclasses of planar graphs, such as pairs of paths, pairs of cycles, and pairs of caterpillars, admit a simultaneous embedding with mapping, while there exist pairs of outerplanar graphs and triples of paths that do not [4]. Recently, it was shown that pairs of trees do not always have such embeddings [9]. Fewer results are known for the less restricted version of the problem where the mapping is not predefined. While it is possible to simultaneously embed without mapping any planar graph with any number of outerplanar graphs, it is not known whether any pair of planar graphs can be simultaneously embedded without mapping [4].

Simultaneous embedding is related to universal pointsets, graph thickness, and geometric thickness. While de Fraysseix et al. [6] showed that there does not exist a universal pointset of size $n$ in the plane for $n$-vertex planar graphs, Bose [3] showed that a set of $n$ points in general position is a universal pointset for trees and outerplanar graphs. Using simultaneous embedding techniques, Duncan et al. [8] showed that degree-four graphs have geometric thickness two.

As we show, colored simultaneous embeddings allow us to generalize the problems above so that the versions with and without mappings become special cases. Formally, the problem of colored simultaneous embedding is defined as follows. The input is a set of planar graphs $G_{1}=\left(V, E_{1}\right)$, $G_{2}=\left(V, E_{2}\right), \ldots, G_{r}=\left(V, E_{r}\right)$ on the same vertex set $V$ and a partition of $V$ into $k$ classes, which we refer to as colors. The goal is to find plane 
straight-line drawings $D_{i}$ of $G_{i}$ using the same $|V|$ points in the plane for all $i=1, \ldots, r$, where vertices mapped to the same point are required to be of the same color.

We call such graphs $k$-colored graphs. Given the above definition, simultaneous embeddings with and without mapping correspond to colored simultaneous embeddings with $k=|V|$ and $k=1$, respectively. Thus, when a set of input graphs allows for a simultaneous embedding without mapping but does not allow for a simultaneous embedding with mapping, there must be a threshold for the number of colors beyond which the graphs can no longer be embedded simultaneously.

In this paper we present the first results about colored simultaneous embeddings. We study different values of $k$ and show that any lineseparated set of points of size $n$ is a universal pointset for $n$-vertex 2 -colored paths. Moreover, there exists a universal pointset of size $n$ for $n$-vertex 3 -colored paths while there is no such universal pointset $n$-vertex 5 -colored paths. We also show how to simultaneously embed a 2-colored outerplanar graph and any number of 2-colored paths. Finally we show the existence of three 6-colored cycles (or four 6-colored paths, or three 9-colored paths) that cannot be simultaneously embedded.

\section{Two-Colored Simultaneous Embeddings}

We begin by showing the existence of a universal pointset for 2-colored paths. The following lemma extends a result of Abellanas et al. [1] on proper 2-colorings of paths.

Lemma 1. Given a 2-colored path $P$ of $r$ red and $b$ blue vertices and a set $S$ of $r$ red and $b$ blue points separated by a line and in general position, there exists a planar straight-line embedding of $P$ into $S$.

Proof. Without loss of generality we can assume that $S$ is separated by a vertical line, and that the red points are on the left of that line. Let $P=v_{0}, v_{1}, \ldots v_{n}$ and let $P_{i}$ be the drawing of the path after the first $i$ vertices of $P$ have been embedded. Let $H_{i}$ be the lower convex envelope of the points of $S$ not used by $P_{i}$. We maintain the following invariants for all $i=0, \ldots, n-1$ for which the colors of $v_{i}$ and $v_{i+1}$ are different:

1. The drawing of $P_{i}$ does not intersect $H_{i}$.

2. The point $p_{i}$ into which the most recent vertex $v_{i}$ has been embedded can see a point of $H_{i}$ of the other color and $P_{i}$ does not intersect the area bounded by this line of sight and the vertical line from $p_{i}$ upward. 


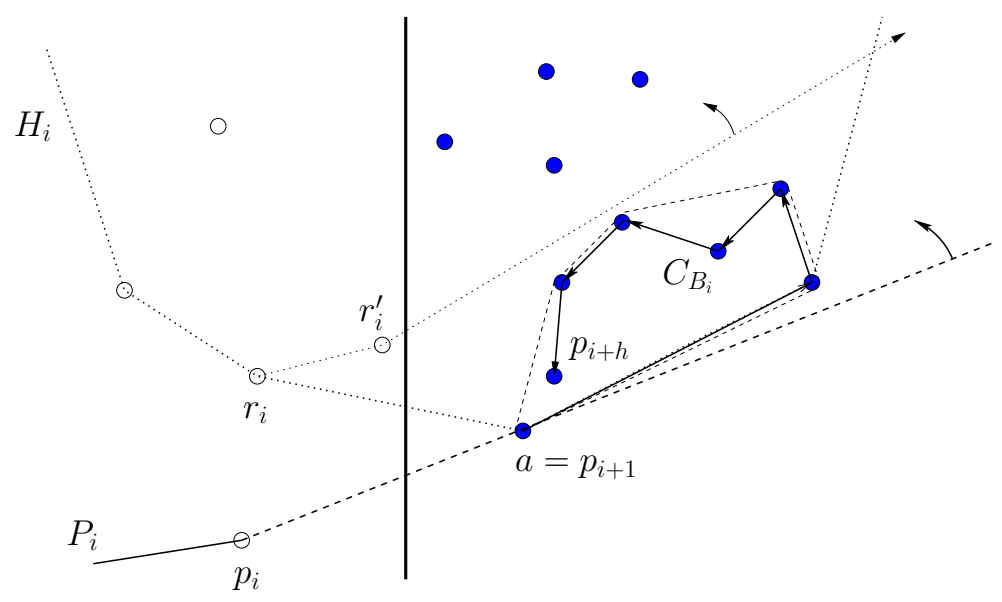

Fig. 1. Embedding a 2-colored path.

Assume that $v_{i}$ is of different color than $v_{i+1}$ and let $h, 1 \leq h \leq n-i$, be maximal such that $v_{i+1}, v_{i+2}, \ldots v_{i+h}$ all have the same color. To maintain the above invariants, we find a line that cuts off the required number $h$ of points of color different from $v_{i}$ from $H_{i}$ (identified with the area on and above it). Assume $v_{i}$ is red (which implies that it has been placed at a point $p_{i}$ in the left half-plane) and $v_{i+1}$ is blue; see Fig. 1.

Consider now the red end-point $r_{i}$ of the unique edge of $H_{i}$ that crosses the vertical separation line. We rotate a ray emanating from $r_{i}$ counterclockwise until either $h$ unused blue points are encountered, or a red point $r_{i}^{\prime}$ lies on the ray. In the latter case, we continue by rotating counterclockwise the ray around $r_{i}^{\prime}$. We repeat this process until $h$ blue points are found, and let $B_{i}$ be the set of identified blue points. Let $C_{B_{i}}$ be the convex hull of $B_{i}$. These points can be added to the path, as follows: Let $a$ be the first blue point of $H_{i}$ that is hit by a ray emanating from $p_{i}$ and rotated counterclockwise. Point $a$ also belongs to $C_{B_{i}}$. We can then connect $p_{i}$ to point $a$. From point $a$ we move counterclockwise along $C_{B_{i}}$ until the right-most point of $C_{B_{i}}$ is reached, while adding each encountered point to the drawing of the path. The remaining points of $B_{i}$ are taken in decreasing value of their $x$-coordinates until the final point, $p_{i+h}$.

The resulting path ending at $p_{i+h}$ satisfies the invariants: $P_{i+h}$ does not intersect $H_{i+h}$ and since $p_{i+h}$ is the leftmost point of $B_{i}$ the second invariant is also satisfied.

Using Lemma 1 we can embed $k$ 2-colored paths for any $k>0$ on a set of 2-colored points in general position in the plane that are separated by a straight-line, provided we have sufficient number of points of each 
color. The resulting set of points is a universal one for these $k 2$-colored paths, which yields the following theorem:

Theorem 1. Any number of 2-colored paths can be simultaneously embedded.

\subsection{A Tree and Paths on Two Colors}

We first show that it is always possible to draw a 2-colored tree in such a way that the two colors are separated by a line.

Lemma 2. Any 2-colored tree can be embedded so that the colors are separated by a straight line.

Proof. We use a divide-and-conquer approach and recursively process the tree from an arbitrary root node. We begin by drawing a vertical line $l$ and assigning the left side to color 1 and the right side to color 2. Next we sort the children of the root by their colors. Let $j$ of the children have color 1 and $k$ children have color 2 .

We can assume without loss of generality that the root is of color 1 and can place it on the left side of line $l$. The $j$ children of color 1 are placed consecutively, such that the first is strictly beneath and to the left of the root, the second is strictly beneath and to the left of the first, and so on. We place the $k$ children of color 2 to the right of line $l$ in a similar fashion. We place the first child strictly beneath and to the right of the root, the second strictly beneath and to the right of the first, and so on. Note that every child has unobstructed line of sight to an horizontal sliver of the plane on both sides of line $l$. Thus, we can recursively place the children of the $j+k$ vertices until the entire tree has been processed.

Now using the result from Lemma 2 we can embed a 2-colored tree on a set of 2-colored points in the plane that are separated by a straight-line. Then we can perturb the positions of the vertices until they are in general position. This can be done without introducing crossings as shown in [4]. From Lemma 1, the resulting set of points is a universal one for 2-colored paths. Together these two results yield the next theorem:

Theorem 2. A 2-colored tree and any number of 2-colored paths can be simultaneously embedded.

\subsection{Planar Graph and Paths on Two Colors}

We have seen that in order to simultaneously embed a 2-colored planar graph $G$ with any number of 2 -colored paths it suffices to find a plane 
drawing of $G$ in which the vertex sets of the same color, $V_{1}$ and $V_{2}$, can be separated by a line. Let $G_{1}$ and $G_{2}$ be the two subgraphs induced by the vertex sets $V_{1}$ and $V_{2}$ respectively. We call such a partition a bipartition, and the edges with vertices from both graphs are called bipartition edges.

Next we present a characterization of the class of 2-colored planar graphs that can be separated by a line. We make extensive use of the characterization and the embedding algorithm for $\mathrm{HH}$ layouts by Biedl et al. [2]. An HH layout is a drawing of a planar bipartition without crossings (but not necessarily using straight-line edges), in which the two vertex sets are separated by a horizontal line. We begin with the characterization of planar bipartitions that can be drawn as HH layouts.

Lemma 3. [2] Planar bipartitions can be realized as HH layouts only if the subgraph $D$ of the dual graph induced by the dual edges of the bipartition edges is connected.

Moreover, it is shown in [2] that $D$ is Eulerian and that it is possible to construct $y$-monotone HH layouts with few bends in linear time. The construction is roughly as follows. Find an Eulerian circuit of $D$ that separates the sets $V_{1}$ and $V_{2}$. Then dummy vertices, that will become bends later, are introduced along the bipartition edges. Next the chain of dummy vertices is processed in the order of the Eulerian circuit and the straight-line drawing algorithm of Chrobak and Kant [5] is applied to the two subgraphs separately by placing one of them below (without loss of generality, say, $G_{1}$ ) and the other above the chain. The final result is straight-line planar drawing with the exception of the bipartition edges which have exactly one bend each; see Fig. 2(a).

This approach does not produce exactly the result that we need. We now show how to obtain a drawing with no bends, while not introducing any crossings, after applying the above technique to the planar bipartition and obtaining the HH layout (which may have some bends).

Lemma 4. From each HH layout with some bends on the separation line, we can derive a straight-line drawing, while keeping the two partitions separated by a line.

Proof. We begin by directing all the edges upward with respect to the basic $\mathrm{HH}$ layout $L$ in order to obtain an upward planar embedding $E$ of $G$. A theorem of Di Battista and Tamassia [7] states that the upward planar embedding $E$ can be realized as a straight-line upward drawing. The resulting drawing, however, may not separate the two sets by a straight horizontal line. Below we show how to obtain the needed straight-line drawing in which the two sets are indeed separable by a line. 


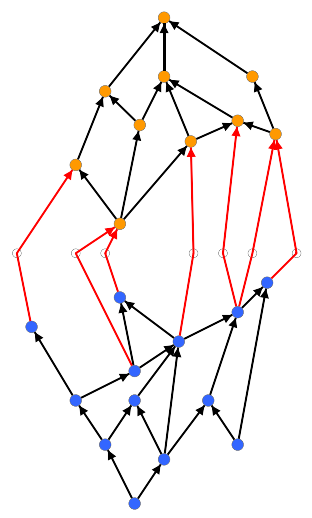

(a) Sample $\mathrm{HH}$ layout.

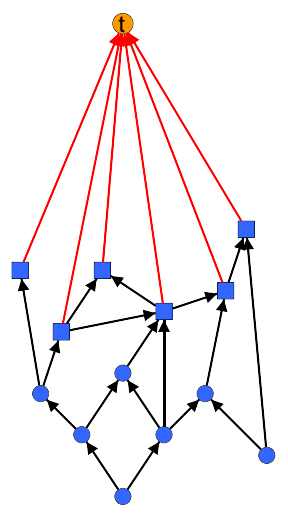

(b) Extended $\mathrm{HH}$ layout of $G_{1}$.

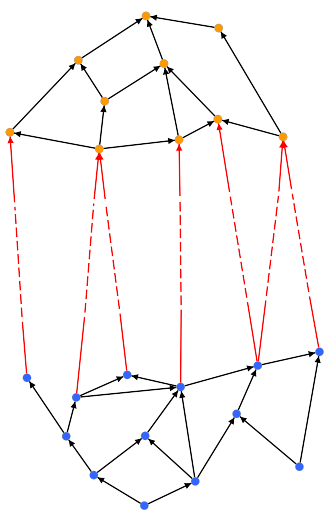

(c) Resulting embedding.

Fig. 2. HH Layouts.

Let $\Gamma_{1}$ be the upward embedding of the graph $G_{1}$ with an upper boundary $B_{1}$ made of vertices adjacent to the bipartition edges. We extend $\Gamma_{1}$ by adding a top vertex $t$ which we connect to all the boundary vertices by edges $(v, t)$, where $v \in B_{1}$. Now we can apply the straight line drawing algorithm of Di Battista and Tamassia to the extended embedding and obtain an upward straight-line drawing, with the vertices on the boundary $B_{1}$ drawn with increasing $x$-coordinates; see Fig 2(b). After removing vertex $t, B_{1}$ is once again the upper boundary. Similarly, we can extend the embedding $\Gamma_{2}$ of $G_{2}$ in order to obtain a drawing with $x$-monotone lower boundary $B_{2}$.

Next we stretch the two layouts in the $x$-direction so that the slopes of the boundary edges become smaller. In particular, we stretch the layouts until all slopes are less than $40^{\circ}$. Note that stretching preserves both planarity and upwardness of the layouts.

Finally we place the two layouts of $\Gamma_{1}$ and $\Gamma_{2}$ above each other and at vertical distance twice the larger of their widths. Now we can safely insert the bipartition edges which connect the two boundaries $B_{1}$ and $B_{2}$. By the choice of separation distance, the slopes of the bipartition edges are larger than $60^{\circ}$. Thus the bipartition edges cannot introduce any crossings and now the two parts can be separated by an horizontal line as desired; see Fig. 2(c).

Lemma 1 and the algorithm above yield the following lemma:

Lemma 5. Let $G$ be a planar bipartition graph in which the dual graph of the subgraph induced by the bipartition edges is connected. (a) Then a straight-line drawing for $G$ can be constructed where the two parts are sep- 
arated by a horizontal line. (b) Since the bipartition includes a 2-coloring, $G$ plus any number of 2-colored paths can be simultaneously embedded.

As 2-colored outerplanar graphs fulfill the conditions of Lemma 5, we have the following theorem:

Theorem 3. A 2-colored outerplanar graph and any number of 2-colored paths can be simultaneously embedded.

\section{$3 \quad k$-Colored Simultaneous Embeddings}

In this section we extend the investigation to more than two colors. We recall that there exist three paths which do not admit a simultaneous embedding with mapping [4], whereas it is easy to see that any number of paths have a simultaneous embedding without mapping. Now we consider $k$-colored paths and/or $k$-colored $k$-cycles for $3 \leq k \leq 9$.

\subsection{Three Colors}

As in the case of 2-colored embeddings we are looking for a universal pointset for paths. A slight modifications of the original universal pointset for 2-colored paths allows us to extend its utility to the 3-colored case.

Theorem 4. Any number of 3-colored paths can be simultaneously embedded.

Proof. Let $P$ be any 3 -colored path with $c_{1}$ vertices of color $1, c_{2}$ vertices of color 2 and $c_{3}$ vertices of color 3 , where $c_{1}+c_{2}+c_{3}=n$. Let $l_{1}, l_{2}$ and $l_{3}$ be three line-segments with a common endpoint $O$ and meeting at $120^{\circ}$ angle. Place $c_{1}$ points along $l_{1}, c_{2}$ points along $l_{2}$, and $c_{3}$ points along $l_{3}$, ensuring that the origin $O$ is not used.

Next map every vertex of the path, in order, to the point of the corresponding color that is closest to the origin and is not already taken. Since every point has line of sight to any other point and for a given $p_{i}$ of $P$ the previous path only blocks line of sight to the points already taken, the result is a plane drawing.

\subsection{Four and Five Colors}

While universal pointsets exist for 1-colored paths, 2-colored paths and 3 -colored paths, we have not been able to find one for 4-colored paths. However, we can show that for $k>4$ universal pointsets for $k$-colored paths do not exist.

Theorem 5. There does not exist a universal pointset for 5-colored paths. 


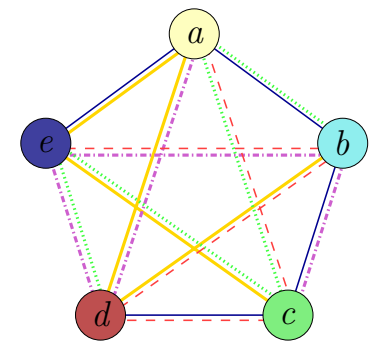

(a) Five 5-colored paths

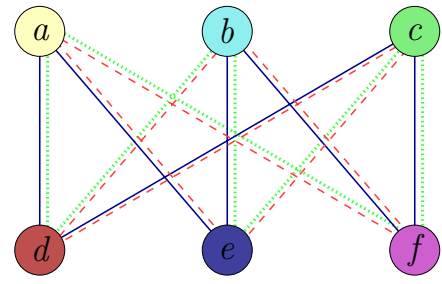

(b) Three 6-colored 6-cycles

Fig. 3. Sets of $k$-colored graphs for $k \in\{5,6\}$ on distinctly colored points whose unions form a $K_{5}$ and a $K_{3,3}$.

Proof. Consider the following five 5 -colored paths on 5 points given in Fig. 3(a) whose union is $K_{5}$ where each edge in the $K_{5}$ belongs to exactly two paths:

1. $a-c-d-b-e$ (thin red dashed edges),

2. $a-d-e-b-c$ (thick light purple alternating dash and dot edges),

3. $b-a-c-e-d$ (thick green dotted edges),

4. $b-d-a-e-c$ (thick yellow solid edges), and

5. $e-a-b-c-d$ (thin blue solid edges).

In any drawing of $K_{5}$ there must be at least one crossing. If this crossing is formed by a pair of edges from different paths then a simultaneous embedding might be possible. However, the paths above were chosen in such a way that every pair of edges either belongs to the same path or is incident. As straight-line incident edges cannot form the crossing pair it suffices to examine all pairs of non-adjacent edges in order to verify that they occur in at least one of the paths.

\subsection{Six and Nine Colors}

Here we consider sets of graphs on pointsets of six or more colors, in which the sets of graphs to simultaneously embed have cardinality less than five.

Lemma 6. There exist three 6-colored cycles that cannot be simultaneously embedded.

Proof. Consider the following three cycles, also shown in Fig. 3(b):

1. $e-a-d-c-f-b-e$ (thin blue solid edges),

2. $e-a-f-b-d-c-e$ (thin red dashed edges), and

3. $a-f-c-e-b-d-a$ (thick green dotted edges). 


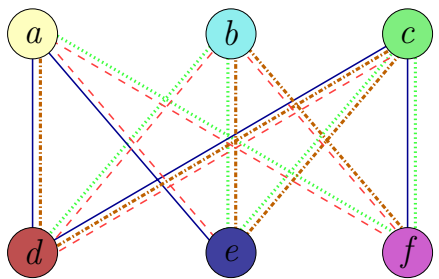

(a) One 5-colored and three 6-colored paths

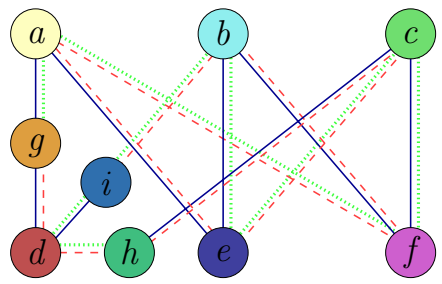

(b) Three 9-colored paths

Fig. 4. Sets of $k$-colored graphs for $k \in\{6,9\}$ on distinctly colored points whose unions form a $K_{3,3}$ or a subdivision thereof.

A visual examination of Fig. 3(b) shows that the union of these cycles forms a $K_{3,3}$. Moreover, every edge in the $K_{3,3}$ belongs to two of the three cycles. In any drawing of $K_{3,3}$ there must be at least one crossing. Since there are only three paths altogether, every pair of edges in the $K_{3,3}$ must share a common 6-cycle, which forces a self-intersecting cycle.

Lemma 7. There exist four 6-colored paths that cannot be simultaneously embedded.

Proof. Fig. 4(a) depicts the following set of one 5-colored path and three 6 -colored paths whose union forms $K_{3,3}$ :

1. $e-a-d-c-f$ (thin blue solid edges),

2. $e-a-f-b-d-c$ (thin red dashed edges),

3. $a-f-c-e-b-d$ (thick green dotted edges), and

4. $a-d-c-e-b-f$ (thick brown dash-and-dots edges).

Every edge in $K_{3,3}$ belongs to at least two of the four paths. As a result, since there are more than three paths, it is easy to manually inspect all 18 pairs of non-adjacent edges to verify that each pair shares a common path. Thus at least one of the paths must be self-intersecting.

Lemma 8. There exist three 9-colored paths that cannot be simultaneously embedded.

Proof. Fig. 4(b) shows that every edge in the subdivided $K_{3,3}$ union belongs to exactly two of the following three paths:

1. $h-c-f-b-e-a-g-d-i$ (thin blue solid edges),

2. $g-d-h-c-e-a-f-b-i$ (thin red dashed edges), and

3. $g-a-f-c-e-b-i-d-h$ (thick green dotted edges).

Since there are only three 9-colored paths altogether, every pair of edges in the subdivided $K_{3,3}$ must share a common path forcing a selfintersecting path. Note that this result is a simplified version of Theorem 2 of Brass et al. [4]. 


\section{Conclusions and Open Problems}

Table 1 summarizes the current status of the newly formulated problem of colored simultaneous embedding. A " $\checkmark "$ indicates that it is always possible to simultaneously embed the type of graphs, a " $\boldsymbol{X}$ " indicates that it is not always possible, and a "?" indicates an open problem.

\begin{tabular}{|c|c|c|c|c|c|c|c|c|}
\hline & $k=1$ & $k=2$ & $k=3$ & $k=4$ & $k=5$ & $k=6$ & $k=9$ & $k=n$ \\
\hline Paths $P_{1} \ldots P_{3}$ & $\checkmark$ & $\checkmark$ & $\checkmark$ & $?$ & $?$ & $?$ & $\boldsymbol{X}$ & $\boldsymbol{X}$ \\
\hline Paths $P_{1} \ldots P_{4}$ & $\checkmark$ & $\checkmark$ & $\checkmark$ & $?$ & $?$ & $\boldsymbol{X}$ & $\boldsymbol{X}$ & $\boldsymbol{X}$ \\
\hline Any number of paths & $\checkmark$ & $\checkmark$ & $\checkmark$ & $?$ & $\boldsymbol{X}$ & $\boldsymbol{X}$ & $\boldsymbol{X}$ & $\boldsymbol{X}$ \\
\hline Planar Graph $G$ and Path $P$ & $\checkmark$ & $\checkmark$ & $?$ & $?$ & $?$ & $?$ & $\boldsymbol{X}$ & $\boldsymbol{X}$ \\
\hline Outerplanar Graph $G$ and Path $P$ & $\checkmark$ & $\checkmark$ & $?$ & $?$ & $?$ & $?$ & $?$ & $?$ \\
\hline Tree $T$ and Path $P$ & $\checkmark$ & $\checkmark$ & $?$ & $?$ & $?$ & $?$ & $?$ & $?$ \\
\hline Two trees $T_{1}, T_{2}$ & $\checkmark$ & $?$ & $?$ & $?$ & $?$ & $?$ & $?$ & $\boldsymbol{X}$ \\
\hline Two planar graphs $G_{1}, G_{2}$ & $?$ & $?$ & $?$ & $?$ & $?$ & $?$ & $\boldsymbol{X}$ & $\boldsymbol{X}$ \\
\hline
\end{tabular}

Table 1. $k$-colored simultaneous embeddings: results and open problems.

\section{References}

1. M. Abellanas, J. García, G. Hernández, M. Noy, and P. Ramos. Bipartite embeddings of trees in the plane. Discrete Applied Math., 93(2-3):141-148, 1999.

2. T. Biedl, M. Kaufmann, and P. Mutzel. Drawing planar partitions: HH-drawings. In 24th Workshop on Graph-Theoretic Concepts in Computer Science (WG), pages 124-136. 1998.

3. P. Bose. On embedding an outer-planar graph in a point set. Computational Geometry: Theory and Applications, 23(3):303-312, 2002.

4. P. Brass, E. Cenek, C. A. Duncan, A. Efrat, C. Erten, D. Ismailescu, S. G. Kobourov, A. Lubiw, and J. S. B. Mitchell. On simultaneous graph embedding. In 8th Workshop on Algorithms and Data Structures (WADS), pages 243-255, 2003.

5. M. Chrobak and G. Kant. Convex grid drawings of 3-connected planar graphs. Intl. Journal of Computational Geometry and Applications, 7(3):211-223, 1997.

6. H. de Fraysseix, J. Pach, and R. Pollack. How to draw a planar graph on a grid. Combinatorica, 10(1):41-51, 1990.

7. G. Di Battista and R. Tamassia. Algorithms for plane representation of acyclic digraphs. Theoretical Computer Science, 61(2-3):175-198, 1988.

8. C. A. Duncan, D. Eppstein, and S. G. Kobourov. The geometric thickness of low degree graphs. In 20th Annual ACM-SIAM Symposium on Computational Geometry (SCG), pages 340-346, 2004.

9. M. Kaufmann, I. Vrťo, and M. Geyer. Two trees which are self-intersecting when drawn simultaneously. In 13th Symposium on Graph Drawing (GD), pages 201-210, 2005. 